\title{
Optimal Training Signals for MIMO OFDM Channel Estimation
}

\author{
Hlaing Minn*, Member, IEEE and Naofal Al-Dhahir, Senior Member, IEEE \\ Department of Electrical Engineering, University of Texas at Dallas \\ hlaing.minn@utdallas.edu, aldhahir@utdallas.edu
}

\begin{abstract}
This paper presents general classes of optimal training signals for estimation of frequency-selective channels in MIMO OFDM systems. Basic properties of the discrete Fourier transform are used to derive the optimal training signals which minimize the channel estimation mean square error. Both single and multiple OFDM training symbols are considered. Several optimal pilot tone allocations among the transmit antennas are presented and classified as frequency division multiplexing, time division multiplexing, code division multiplexing in frequencydomain, code division multiplexing in time-domain, and combinations thereof. All existing optimal training signals in the literature are special cases of the presented optimal training signals and our designs can be applied to pilot-only schemes as well as pilot-data multiplexed schemes.
\end{abstract}

Index Terms-Training signal design, Pilot tone allocation, Channel estimation, MIMO, OFDM, DFT.

\section{INTRODUCTION}

Channel estimation is a critical component in many wireless communications systems. Training-signal-based estimation is especially common in packet-based communications. For single-carrier systems, optimal periodic or aperiodic sequences for channel estimation were studied in [1]-[4] and references therein. Several Optimal designs, placement and energy allocation of training symbols or pilot tones for frequency-selective block-fading channel estimation in single-carrier or/and OFDM systems were recently proposed in [5]-[11]. [6][9] considered training design for SISO single-carrier systems and [7] studied for SISO and MIMO single-carrier systems while [10] presented space-time coded training signal designs in singlecarrier systems. [5][6][8] addressed for SISO OFDM systems and [11][12] studied for MIMO OFDM systems. The training designs are based on minimizing estimation mean square error (MSE) [5] [8] [12] [11] or minimizing Cramer-Rao lower bound [7] or maximizing a lower bound on training-based capacity [6] [9].

In this paper, we revisit the problem of optimal training signal design for frequency-selective block-fading channel estimation in MIMO OFDM systems and present more general optimal training signals based on minimizing the channel estimation MSE. Our results are obtained for the scheme where all sub-carriers are used as pilot tones over $Q$ OFDM symbols. The corresponding results for pilot-data multiplexed schemes are straight-forwardly obtained by replacing the pilot tones of some antennas having FDM pilot allocation with data. All existing optimal training signal designs for MIMO OFDM block-

*Contact Author fading channel estimation can be expressed as special cases of our design.

In Section II, the signal model and the optimality condition for the training signals of multi-transmit-antennas are described. Section III presents optimal training signal design over one OFDM symbol while Section IV generalizes to the case of $Q$ OFDM symbols. In Section V, the applicability of the results to pilot-data multiplexed schemes and the relationships to the existing optimal training signals are discussed. Finally, conclusions are given in Section VI.

\section{Signal Model and Optimality Condition}

Consider a MIMO OFDM system with $K$ sub-carriers and $N_{\text {Tx }}$ transmit-antennas. Since the same channel estimation is performed at each receive antenna, we only need to consider $N_{\text {Tx }}$ transmit antennas and one receive antenna in designing optimal training signals for the channel estimation of MIMO OFDM systems. Consider a system where training signals from $N_{\text {Tx }}$ transmit-antennas are transmitted over $Q$ OFDM symbols. The channel impulse response (CIR) for each transmit-receive antenna pair (including filters' effects) is assumed to have $L$ taps, and is quasi-static over $Q$ OFDM symbols. Let $\boldsymbol{C}_{n, q}$ $=\left[c_{n, q}[0], \ldots, c_{n, q}[K-1]\right]^{T}$ be the pilot tones vector of the $n$-th transmit-antenna at the $q$-th symbol interval and $\left\{s_{n, q}[k]\right.$ : $\left.k=-N_{g}, \ldots, K-1\right\}$ be the corresponding time-domain complex baseband training samples, including $N_{g}(\geq L-1)$ cyclic prefix samples. Define $\boldsymbol{S}_{n}[q]$ as the training signal matrix of size $K \times L$ for the $n$-th transmit-antenna at the $q$-th symbol interval whose elements are given by $\left[\boldsymbol{S}_{n}[q]\right]_{m, l}=s_{n, q}[l-m]$, $m \in\{0, \ldots, K-1\}, l \in\{0, \ldots, L-1\}$.

Let $\boldsymbol{s}_{n, q}$ represent the 0 -th column of $\boldsymbol{S}_{n}[q]$. Then the $l$-th column of $\boldsymbol{S}_{n}[q]$ is the $l$-sample cyclic-shifted version of $\boldsymbol{s}_{n, q}$ denoted by $\boldsymbol{s}_{n, q}^{((l))}$. Assume that $K=M L_{0}$ where $M=1,2, \ldots$, and $L_{0} \geq L$. Let $\boldsymbol{h}_{n}$ denote the length- $L$ CIR vector corresponding to the $n$-th transmit antenna. After the cyclic prefix removal at the receiver, denote the received vector of length $K$ at the $q$-th symbol interval by $\boldsymbol{r}_{q}$. Then the received vector over the $Q$ symbol-intervals is given by

$$
r=S h+n
$$

where

$$
\begin{aligned}
& \boldsymbol{r}=\left[\begin{array}{lll}
\boldsymbol{r}_{0}^{T} & \boldsymbol{r}_{1}^{T} \ldots, \boldsymbol{r}_{Q-1}^{T}
\end{array}\right]^{T} \\
& \boldsymbol{S}=\left[\begin{array}{llll}
\boldsymbol{S}_{0}[0] & \boldsymbol{S}_{1}[0] & \ldots & \boldsymbol{S}_{N_{\mathrm{Tx}}-1}[0] \\
\boldsymbol{S}_{0}[1] & \boldsymbol{S}_{1}[1] & \ldots & \boldsymbol{S}_{N_{\mathrm{Tx}}-1}[1] \\
\vdots & \vdots & \ddots, \vdots & \\
\boldsymbol{S}_{0}[Q-1] & \boldsymbol{S}_{1}[Q-1] & \ldots & \boldsymbol{S}_{N_{\mathrm{Tx}}-1}[Q-1]
\end{array}\right] \\
& \boldsymbol{h}=\left[\boldsymbol{h}_{0}^{T} \boldsymbol{h}_{1}^{T} \ldots \boldsymbol{h}_{N_{\mathrm{Tx}}-1}^{T}\right]^{T} \text {, }
\end{aligned}
$$


and $\boldsymbol{n}$ is a length $K Q$ vector of zero-mean, circularly symmetric, uncorrelated complex Gaussian noise samples with equal variance of $\sigma_{n}^{2}$.

The least-square channel estimate (also maximum likelihood), assuming $\boldsymbol{S}^{H} \boldsymbol{S}$ has full rank, is given by [13]

$$
\hat{\boldsymbol{h}}=\left(\boldsymbol{S}^{H} \boldsymbol{S}\right)^{-1} \boldsymbol{S}^{H} \boldsymbol{r}
$$

and the corresponding MSE is given by $\sigma_{n}^{2} \operatorname{tr}\left\{\left(\boldsymbol{S}^{H} \boldsymbol{S}\right)^{-1}\right\}$. Let $\lambda_{1}, \ldots, \lambda_{L N_{\text {Tx }}}$ be the eigen values (positive) of $\boldsymbol{S}^{H} \boldsymbol{S}$. Then $\operatorname{tr}\left\{\left(\boldsymbol{S}^{H} \boldsymbol{S}\right)^{-1}\right\}=\lambda_{1}^{-1}+\ldots+\lambda_{L N_{\mathrm{Tx}}}^{-1}$. Since $\operatorname{tr}\left\{\left(\boldsymbol{S}^{H} \boldsymbol{S}\right)\right\}=\lambda_{1}+$ $\ldots+\lambda_{L N_{\mathrm{Tx}}}=L \sum_{n=0}^{N_{\mathrm{Tx}}-1} E_{n}$ is a constant, the minimum MSE is achieved iff $\lambda_{1}=\ldots,=\lambda_{L N_{\mathrm{Tx}}}=\frac{1}{N_{\mathrm{Tx}}} \sum_{n=0}^{N_{\mathrm{Tx}}-1} E_{n}=E_{\mathrm{av}}$. This is achieved when

$$
\begin{aligned}
\boldsymbol{S}^{H} \boldsymbol{S} & =E_{\mathrm{av}} \boldsymbol{I} \\
\text { where } \quad E_{\mathrm{av}} & =\frac{1}{N_{\mathrm{Tx}}} \sum_{n=0}^{N_{\mathrm{Tx}}-1} E_{n} \\
E_{n} & =\sum_{q=0}^{Q-1} \sum_{k=0}^{K-1}\left|s_{n, q}[k]\right|^{2} .
\end{aligned}
$$

The corresponding minimum MSE is $L N_{\mathrm{Tx}} \sigma_{n}^{2} / E_{\mathrm{av}}$. We will design training signals for $N_{\mathrm{Tx}}$ transmit antennas to achieve the minimum MSE, i.e. to satisfy the condition (6). The condition (6) can be equivalently stated as

$$
\begin{aligned}
& \text { Condition }-A: \sum_{q=0}^{Q-1} \boldsymbol{S}_{i}^{H}[q] \boldsymbol{S}_{i}[q]=E_{\mathrm{av}} \boldsymbol{I}, \forall i \\
& \text { Condition }-B: \sum_{q=0}^{Q-1} \boldsymbol{S}_{i}^{H}[q] \boldsymbol{S}_{j}[q]=\mathbf{0}, \forall i \neq j .
\end{aligned}
$$

\section{Optimal Training Signal Design over One OFDM SYMBOL}

This section discusses optimal training signal design when training signals from all transmit-antennas are transmitted over only one OFDM symbol. For notational simplicity, the symbol index $q$ will be omitted in this section. For completeness, in the following we summarize the main DFT properties used in this paper. Let $X[n]=\sum_{k=0}^{K-1} x[k] e^{-j 2 \pi k n / K}$ and $x[k]=\frac{1}{K} \sum_{n=0}^{K-1} X[n] e^{j 2 \pi k n / K}$, i.e. $X[n] \stackrel{\mathcal{F}}{\longleftrightarrow} x[k]$.

Property-1: For any $K$, if $X[n]=a, \forall n$, where $a \in \mathbb{C}, \mathbb{C}$ is the field of complex numbers, then $x[k]=a \delta[k]$ where $\delta[k]$ is a discrete unit impulse function.

Property-2: For $K=M L_{0}, M=1,2, \ldots$, if

$$
X[n]= \begin{cases}a, & n=m M ; m=0, \ldots, L_{0}-1 ; a \in \mathbb{C} \\ 0, & \text { else }\end{cases}
$$

then

$$
x[k]= \begin{cases}a L_{0} / K, & k=n L_{0} ; n=0, \ldots, M-1 \\ 0, & \text { else. }\end{cases}
$$

Property-3: $X\left[((n-l))_{K}\right] \stackrel{\mathcal{F}}{\longleftrightarrow} e^{j 2 \pi l k / K} x[k]$ where $((\cdot))_{K}$ denotes modulo- $K$ operation, hence representing a cyclic shifted version. Its dual form is given by $x\left[((k-m))_{K}\right] \stackrel{\mathcal{F}}{\longleftrightarrow}$ $e^{-j 2 \pi m n / K} X[n]$.

Now let us consider the Condition-A in (9) with $Q=1$. The following conditions are observed.

(A-i) Condition-(A.1): The full rank condition in (9) means that for every transmit antenna $i$, there must be at least $L$ different nonzero tones.

(A-ii) The condition $\left(\boldsymbol{s}_{n}^{((l))}\right)^{H} \boldsymbol{s}_{n}^{((l))}=E_{\mathrm{av}}$ from (9) means that

$$
\text { Condition-(A.2) : } E_{n}=E_{\text {av }}, \forall n .
$$

(A-iii) Consider the following condition from (9):

$$
\left(\boldsymbol{s}_{n}^{((l))}\right)^{H} \boldsymbol{s}_{n}^{((m))}=0, \forall l \neq m ; l, m \in\{0,1, \ldots, L-1\} .
$$

Since $\boldsymbol{s}_{n}^{((l))}=F_{K}^{-1} W(l) \boldsymbol{C}_{n}$ where $F_{K}$ is the $K$ point FFT matrix and $W(l)=\operatorname{diag}\left\{1, e^{-j 2 \pi l / K}, \ldots, e^{-j 2 \pi(K-1) l / K}\right\}$ is a diagonal matrix, the following condition is obtained from (14):

$$
\begin{aligned}
\text { Condition - (A.3): } \quad & \sum_{k=0}^{K-1}\left|c_{n}[k]\right|^{2} e^{j 2 \pi d k / K}=0, \\
& \text { for } d= \pm 1, \ldots, \pm(L-1) .
\end{aligned}
$$

(A-iii-a) By Property-1, the condition (15) is satisfied for any $K \geq L$ if

$$
\left|c_{n}[k]\right|^{2}=a_{n}, \forall k, a_{n}>0 .
$$

(A-iii-b) By Properties 2 and 3, the condition (15) is satisfied for $K=M L_{0}, M=1,2, \ldots$ and $L_{0} \geq L$, if

$$
\left|c_{n}[k]\right|^{2}= \begin{cases}a_{n}^{(l)}, & k=l+m M ; l=0, \ldots, M-1 ; \\ & m=0, \ldots, L_{0}-1 ; a_{n}^{(l)} \geq 0 \\ 0, & \text { else. }\end{cases}
$$

Let us consider the Condition-B in (10). Using $\boldsymbol{s}_{n}^{((l))}=$ $F_{K}^{-1} W(l) \boldsymbol{C}_{n}$, we obtain the following condition:

$$
\begin{aligned}
& \sum_{m=0}^{K-1} c_{i}^{*}[m] c_{j}[m] e^{j 2 \pi d m / K}=0 \\
& \text { for } d=0, \pm 1, \ldots, \pm(L-1), \forall i \neq j .
\end{aligned}
$$

Let $G_{i, j}[m]=c_{i}^{*}[m] c_{j}[m]$ and $g_{i, j}[n] \stackrel{\mathcal{F}}{\longleftrightarrow} G_{i, j}[m]$. Then the above condition can be expressed as

$$
\begin{aligned}
& \text { Condition }-(B .1): \sum_{m=0}^{K-1} G_{i, j}[m] e^{j 2 \pi d m / K}=0, \\
& \quad \text { for } d=0, \pm 1, \ldots, \pm(L-1), \forall i \neq j \\
& \text { or } \quad g_{i, j}[n]=0, \\
& \quad \text { for } n=0, \ldots, L-1, K-L+1, \ldots, K-1 .
\end{aligned}
$$

(B-i) If $L \leq K<2 L$, then (20) requires that $g_{i, j}[n]=0 \forall n$ and hence, $G_{i, j}[m]=0 \forall m$ which is possible only if pilots from different transmit-antennas are frequency division multiplexed (FDM). However, since each transmit-antenna must have at least $L$ tones according to the Condition-(A.1), we can have only one transmit-antenna, i.e., $N_{\mathrm{Tx}}=1$. The optimal pilot tones are then given by (16) (constant amplitude pilot tones) with $N_{\mathrm{Tx}}=1$. 
(B-ii) If $2 L \leq K<3 L, K=M L_{0}, M=2$, and $L_{0} \geq L$, then $g_{i, j}[n]$ can have nonzero values at indices $\{L, \ldots, K-L\}$ while satisfying (20). Using Properties 2 and 3, we have two solutions which satisfy the condition (19) or (20):

(B-ii-a) All antennas use all pilot tones and have the following relationship:

$$
\begin{aligned}
G_{i, j}[n] & = \begin{cases}a_{i, j}^{(l)}, & n=k_{l}+m M ; l=0,1 \\
& k_{0}=0 ; k_{1}=1 ; m=0, \ldots, L_{0}-1 \\
0, & \text { else }\end{cases} \\
a_{i, j}^{(0)} & =-a_{i, j}^{(1)}, \quad a_{i, j}^{(l)} \in\{\mathbb{C} \backslash 0\}
\end{aligned}
$$

where \denotes the set minus operation. Note that $a_{i, j}^{(0)}=$ $-a_{i, j}^{(1)}$ limits the number of transmit-antennas to $N_{\mathrm{Tx}}=2$. This allocation type will be called code division multiplexing (CDM) pilot allocation.

(B-ii-b) Pilot tones of an antenna are disjoint from those of any other antenna. Since each antenna must have at least $L$ tones, we can have only $N_{\mathrm{Tx}}=2$ antennas and

$$
G_{1,2}[m]=0, \forall m \text {. }
$$

To satisfy the Condition-(A.3) from (15), each antenna's pilots must comply with (17), i.e., they must be spread out with equal spacing over the indices $\{0, \ldots, K-1\}$. Both antennas must have the same number of pilot tones $L_{0}$ since $2 L \leq K<3 L$, $K=2 L_{0}$. To satisfy the Conditions (A.2) from (13) and (A.3) from (15), all pilot amplitudes must be the same. This type of allocation of equally-spaced, disjoint pilot tones in the frequency-domain will be called frequency division multiplexing (FDM) pilot allocation.

(B-iii) In general, consider $M L \leq K<(M+1) L, K=$ $L_{0} M, L_{0} \geq L$ for $M=1,2, \ldots$. Let $L_{1}=L_{0} V, U=M / V$, $V \in\{1,2, \ldots, M\}$. We can have $U$ FDM groups, each has $L_{1}$ pilot tones. For the $u$-th FDM group $(u \in\{0,1, \ldots, U-1\})$, the condition (19) is satisfied if the following two conditions are met:

$$
\begin{aligned}
& \text { Condition - (B.1.1) : } \\
& G_{u V+i, u V+j}[n]= \begin{cases}a_{u V+i, u V+j}^{(l)}, & n=k_{l}^{(u)}+m M ; \\
& l=0, \ldots, V-1 ; \\
& \begin{array}{l}
\forall i \neq j \\
\text { else }
\end{array}\end{cases} \\
& \sum_{l=0}^{V-1} a_{u V+i, u V+j}^{(l)}=0, \forall i \neq j
\end{aligned}
$$

where $k_{l}^{(u)} \neq k_{m}^{(u)}$ if $l \neq m, k_{n}^{(u)} \in\{0, \ldots, M-1\}$, and $a_{u V+i, u V+j}^{(l)} \in \mathbb{C}$. For any $u \neq u^{\prime}, k_{l}^{(u)}$ and $k_{l}^{\left(u^{\prime}\right)}$ are disjoint. The first condition (24) can be satisfied by infinite ways but the second condition (25) can be satisfied by only a few. Hence, (25) determines the number of antennas within an FDM group. If $a_{i, j}^{(l)}$ is restricted to have constant amplitude for any antenna pair $(i, j)$, a simple solution to (25) is given by

$$
\begin{aligned}
a_{u V+i, u V+j}^{(l)}= & a_{u V+i, u V+j}^{(0)} e^{j 2 \pi l(j-i) / V}, \\
& \forall i \neq j, i, j \in\{0, \ldots, V-1\} .
\end{aligned}
$$

This solution indicates that a maximum of only $V$ antennas are possible within each FDM group.
The corresponding optimal pilot tones within each FDM group satisfying the conditions (24)(25) (and hence, the Conditions (B.1) and (A.1)) are then given by

$$
\begin{aligned}
& c_{u V+i}[n]= \begin{cases}b_{u V+i}^{(l)}[m], & n=k_{l}^{(u)}+m M ; \\
& m=0, \ldots, L_{0}-1 ; \\
& l=0, \ldots, V-1 ; \\
& u=0, \ldots, U-1 \\
\text { else }\end{cases} \\
& 0, \quad \begin{array}{c}
i=0, \ldots, V-1 \\
b_{u V+i}^{(l)}[m]=
\end{array} \\
&\left|b_{u V}^{(l)}[m]\right|=\quad b_{0}^{(l)}>0 .
\end{aligned}
$$

It can be readily checked that the solution (27) satisfies (17) and hence, the Condition-(A.3) from (15). Although the condition (25) is satisfied by any $p_{i} \in\{\mathbb{C} \backslash 0\}$, imposing the Condition(A.2) from (13) yields $p_{i}=1$ and hence, it is incorporated in (27). The pilot allocation within each FDM group may be considered as CDM type in the frequency domain and hence, will be called $\operatorname{CDM}(\mathrm{F})$. The overall pilot allocation will be called $U-\mathrm{FDM}+V-\mathrm{CDM}(\mathrm{F})$ pilot allocation. The total number of antennas is $N_{\mathrm{Tx}}=U V=M$. If $U=1$, we have a $\operatorname{CDM}(\mathrm{F})$ pilot allocation while if $V=1$, we have an FDM pilot allocation. If $L \leq K<2 L$, i.e., $M=1$, then $V=1$ (one antenna within an FDM group), $U=1$ (only one FDM group) and the result from (27) becomes that in (B-i). For $2 L \leq K<3 L$, i.e., $M=2$, if $V=1$, then $U=2$ and the result from (27) becomes that in (B-ii-b). If $V=2$, then $U=1$ and the result from (27) becomes that in (B-ii-a).

Note that for $V>1$, all the pilot tones of the corresponding antenna do not have to be equally spaced. The solution (27) suggests that within each FDM group, the pilot amplitude is the same. However, for $V=2$, we can have an alternative solution:

$$
\begin{aligned}
b_{1}^{(l)}[m]= & \frac{1}{b_{0}^{(l) *}[m]}(-1)^{l}, \\
& l=0,1 ; m=0, \ldots, L_{0}-1 \\
\left|b_{0}^{(1)}[m]\right|= & \frac{1}{\left|b_{0}^{(0)}[m]\right|} \\
\left|b_{i}^{(l)}[m]\right|= & a_{i}^{(l)}>0 \text { for } m=0, \ldots, L_{0}-1
\end{aligned}
$$

where $\left\{b_{k}^{(l)}[m]\right\}$ for different $l$ do not necessarily have the same amplitude.

FDM antenna groups may have different numbers of pilot tones, which must be an integer multiple of $L_{0}$. In this case, due to the different numbers of pilot tones per antenna, the corresponding pilot amplitude will be different so that the total pilot energy per antenna is the same (13). More complicated pilot allocations are also possible, for example, by not fixing the FDM boundaries and by combining FDM and CDM types. As an example, Table I presents a set of optimal pilot vectors for an OFDM system with $K=16, L=2, N_{\mathrm{Tx}}=8$, and $Q=1$ where $\alpha_{k}$ can be any constant-modulus symbol. Antennas 0,1 , 2 , and 3 are of FDM type among themselves. Within the group of antennas 4 and 5 or the group of antennas 6 and 7, the pilot allocation is of $\mathrm{CDM}(\mathrm{F})$ type while between the two groups it is of FDM type. Pilot allocation between antenna 4 (or 5) and antenna 0 (or 1 ) is $\mathrm{CDM}(\mathrm{F})$ type, and so it is between antenna 6 (or 7) and antenna 2 (or 3). Between antenna 4 (or 5) and antenna 2 (or 3 ) it is FDM type, and so it is between antenna 
6 (or 7) and antenna 0 (or 1). Note that pilot amplitudes may be different for different antennas, e.g., between antenna 0 and antenna 4.

\section{Optimal Training Signal Design over Multiple OFDM SYMBOLS}

This section considers training signal design for channel estimation based on observations over $Q$ OFDM symbols. Let us consider the Condition-A from (9). The following are observed:

(A-i) Condition-(A.1): The full rank condition in (9) means that for every transmit antenna and within the $Q$ OFDM symbols, there must be at least $L$ different nonzero tones, each with at least one symbol duration.

(A-ii) The condition

$$
\sum_{q=0}^{Q-1} \boldsymbol{s}_{n, q}^{H} \boldsymbol{s}_{n, q}=E_{\mathrm{av}} \boldsymbol{I}, \forall n
$$

means that

$$
\text { Condition-(A.2) : } E_{n}=E_{\mathrm{av}}, \forall n \text {. }
$$

(A-iii) The condition

$$
\sum_{q=0}^{Q-1}\left(\boldsymbol{s}_{n, q}^{((l))}\right)^{H} \boldsymbol{s}_{n, q}^{((m))}=0, \forall l \neq m ; l, m \in\{0,1, \ldots, L-1\}
$$

means that

$$
\sum_{q=0}^{Q-1} \sum_{n=0}^{K-1}\left|c_{k, q}[n]\right|^{2} e^{j 2 \pi d n / K}=0 \text { for } d= \pm 1, \pm 2, \ldots, \pm(L-1) .
$$

By defining

$$
E_{k}[n]=\sum_{q=0}^{Q-1}\left|c_{k, q}[n]\right|^{2}, n=0, \ldots, K-1,
$$

we can express (34) as

$$
\begin{aligned}
& \text { Condition }-(\text { A.3 }): \\
& \sum_{n=0}^{K-1} E_{k}[n] e^{j 2 \pi d n / K}=0 \text { for } d= \pm 1, \pm 2, \ldots, \pm(L-1) .
\end{aligned}
$$

Note that $E_{k}=\sum_{n=0}^{K-1} E_{k}[n]$. Using Properties 2 and 3, we obtain the following condition satisfying (34) for $M L \leq K<$ $(M+1) L, K=M L_{0}$, and $L_{0} \geq L$ :

$$
E_{k}[n]= \begin{cases}a_{k}^{(l)}, & n=l+m M ; l=0, \ldots, M-1 \\ & m=0, \ldots, L_{0}-1 ; a_{k}^{(l)} \geq 0 \\ 0, & \text { else. }\end{cases}
$$

At least one $a_{k}^{(l)}$ must be nonzero in order to get nonzero $E_{k}$. Note that pilot tone amplitudes of different antennas may not be necessarily the same but total pilot energies $E_{k}$ must be the same for different antennas.

Now consider the Condition-B from (10) which is given by

$$
\begin{aligned}
\sum_{q=0}^{Q-1} \sum_{m=0}^{K-1} c_{i, q}^{*}[m] c_{j, q}[m] e^{j 2 \pi d m / K} & =0 \\
\quad \text { for } d=0, \pm 1, \ldots, \pm(L-1) & ; \quad \forall i \neq j .
\end{aligned}
$$

IEEE Communications Society
Let $G_{i, j}[q, m]=c_{i, q}^{*}[m] c_{j, q}[m]$ and $G_{i, j}[q, n] \stackrel{\mathcal{F}}{\longleftrightarrow} g_{i, j}[q, k]$. Then (10) becomes

$$
\begin{aligned}
& \text { Condition }-(B .1): \\
& \sum_{m=0}^{K-1}\left(\sum_{q=0}^{Q-1} G_{i, j}[q, m]\right) e^{j 2 \pi d m / K}=0 \\
& \text { for } d=0, \pm 1, \ldots, \pm(L-1) ; \forall i \neq j \\
\text { or } \quad & \sum_{q=0}^{Q-1} g_{i, j}[q, n]=0, \\
& \text { for } n=0, \ldots, L-1, K-L+1, \ldots, K-1 ; \forall i \neq j .
\end{aligned}
$$

(B-i-a) If we let $g_{i, j}[q, n]=0$, for $n=0, \ldots, L-1, K-L+1$, $\ldots, K-1, \forall i \neq j$ and $\forall q$, then the solution for one OFDM symbol can be directly applied to each symbol of the $Q$ symbol case. To wit, for $M L \leq K<(M+1) L, K=M L_{0}$, and $L_{0} \geq L$, we have $Q$ sets of antennas where each set has $M$ antennas whose pilot tone allocation are defined by the solution for the one-symbol case. Each set uses one symbol out of $Q$ symbols. Within each set, pilot tone allocation is of $\mathrm{CDM}(\mathrm{F})$ or FDM or FDM+CDM(F) type while different sets are of TDM type. Total number of antennas is $N_{\mathrm{Tx}}=M Q$.

(B-i-b) Alternatively, using Properties 2 and 3, we have the following condition satisfying (10) for $M L \leq K<(M+1) L$, $K=M L_{0}$, and $L_{0} \geq L$ :

$\sum_{q=0}^{Q-1} G_{i, j}[q, m]= \begin{cases}a_{i, j}^{(l)}, & m=l+k M, k=0, \ldots, L_{0}-1 \\ & l=0, \ldots, M-1 ; \forall i \neq j \\ 0, & \text { else },\end{cases}$

and $\sum_{l=0}^{M-1} a_{i, j}^{(l)}=0, \forall i \neq j ; a_{i, j}^{(l)} \in \mathbb{C}$.

Note that (41) satisfies $\sum_{q=0}^{Q-1} g_{i, j}[q, n]=0$ for $n=1, \ldots$, $L-1, K-L+1, \ldots, K-1, \forall i \neq j$ while (42) satisfies $\sum_{q=0}^{Q-1} g_{i, j}[q, n]=0$ for $n=0, \forall i \neq j$.

(B-i-b-1) If $G_{i, j}[q, m]=0, \forall q$ and $\forall m$, for some $i \neq j$ (a group of antennas), the corresponding pilot tones are disjoint from each other. Within the group, the $L_{0}$ tones of each antenna are disjoint from those of any other antenna by means of multiplexing in time (TDM), in frequency (FDM) or in both time and frequency (TFDM). The pilot tones must also satisfy the Conditions (A.2) and (A.3) (through (32), (37)). Hence, the optimal $L_{0}$ pilot tones of one symbol duration for each antenna must be equally spaced ( $M$ tone spacing) with equal amplitude. They should be disjoint from pilot tones of any other antenna. All antennas have the same pilot amplitude.

(B-i-b-2) Now, let us consider the case where $G_{i, j}[q, m] \neq 0$, for some (or all) $q$, for some (or all) $m$, and for some $i \neq j$, (a group of antennas where $i, j \in\left\{i_{0}, i_{1}, \ldots\right\}$ ). Let $N_{f}$ be the number of sets of equally spaced ( $M$ tone spacing) $L_{0}$ tones over $Q$ symbols assigned to an antenna from the above group (each set corresponds to tone indices $\left\{k_{l}+m M\right.$ : $\left.m=0, \ldots, L_{0}-1\right\}$ where $k_{l} \neq k_{m}$ if $l \neq m$ and $k_{n} \in$ $\{0, \ldots, M-1\})$. Let $N_{t}$ be the number of repetitions in time (with symbol indices $q_{0}, q_{1}, \ldots, q_{N_{t}-1}$ ) of the above $N_{f}$ sets of $L_{0}$ tones each. By using the same principle as in (26), within the $q$-th symbol interval, the $N_{f}$ sets of pilot tones (each set has $L_{0}$ tones) can accommodate a set of $N_{f}$ antennas if

$$
\begin{aligned}
& a_{i_{m}, i_{n}}^{\left(k_{l}\right)}\left[q_{t}\right]=a_{i_{m}, i_{n}}^{\left(k_{0}\right)}\left[q_{t}\right] e^{j 2 \pi l(n-m) / N_{f}} \\
& \text { for } l=0, \ldots, N_{f}-1 ; m \neq n ; m, n \in\left\{0, \ldots, N_{f}-1\right\} .
\end{aligned}
$$


The corresponding pilot tones are given by

$$
\begin{aligned}
& c_{i_{m}, q_{t}}[n]= \begin{cases}b_{m}^{(l)}\left[q_{t}, d\right], & n=k_{l}+d M \\
& d=0, \ldots, L_{0}-1 \\
& l=0, \ldots, N_{f}-1 \\
0, & \text { else }\end{cases} \\
& b_{m}^{(l)}\left[q_{t}, d\right]=b_{0}^{(l)}\left[q_{t}, d\right] e^{-j 2 \pi l m / N_{f}}, m=0, \ldots, N_{f}-1 \\
& \left|b_{0}^{(l)}\left[q_{t}, d\right]\right|=b_{0}>0 \text {, }
\end{aligned}
$$

where the fulfillment to the Conditions (A.1), (A.2), (A.3), and (B.1) is inherited from (27). This pilot allocation is of CDM(F) type.

Applying the CDM principle over the above $N_{t}$ symbols can accommodate $N_{t}$ sets of $N_{f}$ antennas each. The optimal pilot tones for an antenna from the $v$-th set are related to those from the 0 -th set by

$$
\begin{array}{r}
\left\{a_{i_{v N_{f}+m}, i_{v N_{f}+n}}^{\left(k_{l}\right)}\left[q_{t}\right]: \forall l\right\}=\left\{a_{i_{m}, i_{n}}^{\left(k_{l}\right)}\left[q_{0}\right]: \forall l\right\} e^{j 2 \pi t v / N_{t}}, \\
\text { for } v=0, \ldots, N_{t}-1 ; l=0, \ldots, N_{f}-1 ; \\
\forall m \neq n ; m, n \in\left\{0, \ldots, N_{f}-1\right\} .
\end{array}
$$

The corresponding pilot tones are given by

$$
c_{i_{v N_{f}+m}, q_{t}}[n]=c_{i_{m}, q_{0}}[n] e^{-j 2 \pi t v / N_{t}}, v=0, \ldots, N_{t}-1
$$

and $\left\{c_{i_{m}, q_{0}}[n]: m=0, \ldots, N_{f}-1\right\}$ are given by (44). The fulfillment to the Conditions (A.1), (A.2), (A.3), and (B.1) is inherited from (44). This pilot allocation among the $N_{t}$ sets is of CDM type in the time-domain, $\operatorname{CDM}(\mathrm{T})$. Hence, this overall pilot allocation over $N_{f} L_{0}$ tones and $N_{t}$ symbols may be referred to as $N_{f}-\mathrm{CDM}(\mathrm{F})+N_{t}-\mathrm{CDM}(\mathrm{T})$.

More complicated pilot allocation schemes are also possible by combining FDM, TDM, TFDM, CDM(F), and CDM(T) type allocations. As an example of optimal pilot allocations of mixed types, Table II shows a sample layout of which antenna is assigned to which pilot tone at which symbol for an OFDM system with $K=16, L=4, N_{\mathrm{Tx}}=8$, and $Q=2$. The group of antennas $0,1,2,3$ is disjoint from the others by FDM over 2 symbols. Antenna 4 is of TFDM type while antenna 5 is of purely FDM over one symbol. The group of antennas 6, 7 is disjoint from the others by TFDM. Within the group of antennas $0,1,2,3$ pilot allocation is of $2-\mathrm{CDM}(\mathrm{F})+2-\mathrm{CDM}(\mathrm{T})$ type. Within the group of antennas 6 and 7, pilot allocation is of $\mathrm{CDM}(\mathrm{F})$ type.

In Table III, the pilot vector for each antenna is presented for the optimal training signal structure given in Table II. $\alpha_{k}$ can be any constant-modulus symbol. Note that within one symbol, pilot amplitudes from different antennas may be different, e.g., compare pilot amplitudes of antennas 0,4 , and 6.

\section{Discussion}

Our findings are summarized in the following. For the training signal design over one OFDM symbol, a simple optimal solution is the FDM+CDM(F) type pilot allocation given by (27). More complicated pilot allocations can be constructed by not fixing the FDM boundaries and by combining FDM and $\mathrm{CDM}(\mathrm{F})$. An example is given in Table I. For the training signal design over $Q$ OFDM symbols, two simple optimal solutions have been presented. The first one is composed of $Q$ TDM groups and within each TDM group, the FDM+CDM(F) pilot allocation given in (27) is applied. In the second solution, every antenna transmits on all pilot tones over all $Q$ OFDM symbols and the optimal pilot tones are given by the $\mathrm{CDM}(\mathrm{F})+\mathrm{CDM}(\mathrm{T})$ allocation defined in (46) and (44). Other more complicated solutions can be obtained by combining TDM (less than $Q$ TDM groups), FDM, CDM(F), and CDM(T) allocations. An example is given in Tables II and III.

We have numerically evaluated the optimality condition (6) for all the training signal designs discussed and confirmed their optimality with respect to minimizing MSE.

Although our discussion is based on pilot-only $Q$ OFDM training symbols, the results can be straight-forwardly applied to pilot-data multiplexed schemes by using data in place of pilot tones allocated to some transmit antennas with FDM pilot allocation in the original pilot-only training design (and removing those transmit antennas). The orthogonality between data and pilot tones is inherited from the original FDM pilot allocation.

Let us consider pilot-data multiplexed schemes with $M L \leq$ $K<(M+1) L, K=L_{0} M, L_{0} \geq L$, and $M=\left(M_{d}+M_{p}\right)$, where $M_{d} L_{0}$ sub-carriers are for data and $M_{p} L_{0}$ sub-carriers are for pilot tones. When $K=D M_{p} L_{0}$ where $D$ is an integer $(>1)$ and pilot tones are equal-powered, equal-spaced and all transmit antennas use all pilot tones with $\mathrm{CDM}(\mathrm{F})$ pilot allocation (i.e., $U=1, k_{l}=\tau+l D$ with $\tau \in\{0, \ldots, D-1\}$, $l=0, \ldots, M_{p}-1$, in (27)), our results specialize to the optimal training signals for $M_{p}$ transmit antennas over one OFDM symbol presented in [6] and [11]. Note that $K$ needs not be an integer multiple of $M_{p} L_{0}$ in our designs for pilot-data multiplexed schemes. Our optimal training signal designs over multiple symbols can be similarly linked to those of [11]. Now let us consider schemes where all sub-carriers are pilot tones. When $K=M L_{0}$, and if CDM pilot allocation is used over all $K$ tones, our design becomes identical to the optimal training signal design for $M$ transmit antennas presented in [12].

\section{CONCLUSiOnS}

In this paper, we presented general classes of optimal training signals for channel estimation in MIMO OFDM systems. For the estimation of $L$-tap channel impulse response corresponding to each transmit-receive antenna pair based on $Q$ OFDM training symbols, the optimal training signal for each transmit antenna has $M L_{0}$ different pilot tones of one symbol duration within the $Q$ symbols where $\left(L_{0} \geq L\right), M \in\left\{1,2, \ldots, K / L_{0}\right\}$ and the number of sub-carriers $K$ is an integer multiple of $L_{0}$. The $L_{0}$ pilot tones in each set should be equally spread out with equal spacing over the frequency domain and should have equal total energy over the $Q$ OFDM symbols. The spacing between different sets of $L_{0}$ pilot tones can be chosen arbitrarily as long as the different sets are disjoint. All transmit antennas must have the same total training signal energy over the $Q$ OFDM symbols. The optimal pilot tone allocation among transmit antennas can be of frequency division multiplexing, time division multiplexing, code division multiplexing in time domain, code division multiplexing in frequency domain or combinations thereof. All existing optimal training signals in the literature are special cases of the presented optimal training signals. 
TABLE I

Optimal Pilot TONE VECTORS FOR $N_{\text {Tx }}=8$ TRANSMIT ANTENNAS IN AN OFDM SYSTEM WITH $K=16$ SUB-CARRIERS OVER $Q=1$ OFDM SYMBOL FOR ESTIMATION OF CHANNELS WITH $L=2$ TAPS EACH

\begin{tabular}{||c|c|c|c|c|c|c|c||}
\hline \multirow{2}{*}{0} & 1 & 2 & 3 & 4 & 5 & 6 & 7 \\
\hline$\sqrt{2} \alpha_{1}$ & 0 & 0 & 0 & $\alpha_{1}$ & $\alpha_{1}$ & 0 & 0 \\
0 & $\sqrt{2} \alpha_{3}$ & 0 & 0 & $\alpha_{3}$ & $-\alpha_{3}$ & 0 & 0 \\
0 & 0 & $\sqrt{2} \alpha_{5}$ & 0 & 0 & 0 & $\alpha_{5}$ & $\alpha_{5}$ \\
0 & 0 & 0 & $\sqrt{2} \alpha_{7}$ & 0 & 0 & $\alpha_{7}$ & $-\alpha_{7}$ \\
$\sqrt{2} \alpha_{2}$ & 0 & 0 & 0 & $-\alpha_{2}$ & $-\alpha_{2}$ & 0 & 0 \\
0 & $\sqrt{2} \alpha_{4}$ & 0 & 0 & $-\alpha_{4}$ & $\alpha_{4}$ & 0 & 0 \\
0 & 0 & $\sqrt{2} \alpha_{6}$ & 0 & 0 & 0 & $-\alpha_{6}$ & $-\alpha_{6}$ \\
0 & 0 & 0 & $\sqrt{2} \alpha_{8}$ & 0 & 0 & $-\alpha_{8}$ & $\alpha_{8}$ \\
$\sqrt{2} \alpha_{1}$ & 0 & 0 & 0 & $\alpha_{1}$ & $\alpha_{1}$ & 0 & 0 \\
0 & $\sqrt{2} \alpha_{3}$ & 0 & 0 & $\alpha_{3}$ & $-\alpha_{3}$ & 0 & 0 \\
0 & 0 & $\sqrt{2} \alpha_{5}$ & 0 & 0 & 0 & $\alpha_{5}$ & $\alpha_{5}$ \\
0 & 0 & 0 & $\sqrt{2} \alpha_{7}$ & 0 & 0 & $\alpha_{7}$ & $-\alpha_{7}$ \\
$\sqrt{2} \alpha_{2}$ & 0 & 0 & 0 & $-\alpha_{2}$ & $-\alpha_{2}$ & 0 & 0 \\
0 & $\sqrt{2} \alpha_{4}$ & 0 & 0 & $-\alpha_{4}$ & $\alpha_{4}$ & 0 & 0 \\
0 & 0 & $\sqrt{2} \alpha_{6}$ & 0 & 0 & 0 & $-\alpha_{6}$ & $-\alpha_{6}$ \\
0 & 0 & 0 & $\sqrt{2} \alpha_{8}$ & 0 & 0 & $-\alpha_{8}$ & $\alpha_{8}$ \\
\hline
\end{tabular}

Depending on the pilot allocation, the pilot amplitudes of different antennas within an OFDM symbol can be different and all the pilot tones of an antenna may not be equally spaced. The presented optimal training signal designs are applicable to pilot-only schemes as well as pilot-data multiplexed schemes.

\section{REFERENCES}

[1] A. Milewski, "Periodic sequences with optimal properties for channel estimation and fast start-up equalization," IBM Journal of Research and Development, Vol. 27, No. 5, Sept. 1983, pp. 426-431.

[2] C. Tellambura, M.G. Parker, Y.J. Guo, S.J. Shepherd and S.K. Barton, "Optimal sequences for channel estimation using discrete Fourier transform techniques," IEEE Trans. Commun., Vol. 47, No. 2, Feb. 1999, pp. 230238.

[3] Wai Ho Mow, "A New Unified Construction of Perfect Root-of-Unity Sequences," Proc. Spread Spectrum Techniques and its Applications (ISSSTA'96), Mainz, Germany, pp. 955-959, 1996.

[4] J.C.L. Ng, K.B. Letaief, and R.D. Murch, "Complex Optimal Sequences with Constant Magnitude for Fast Channel Estimation Initialization," IEEE Trans. on Commun., Vol. 46, N0. 3, Mar. 1998, pp. 305-308.

[5] J.H. Manton, "Optimal training sequences and pilot tones for OFDM systems," IEEE Communications Letters, Vol. 5, No. 4, April 2001, pp. 151153.

[6] S. Adireddy, L. Tong, and H. Viswanathan, "Optimal Placement of Training for Frequency-Selective Block-Fading Channels," IEEE Trans. on Information Theory, Vol. 48, No. 8, Aug. 2002, pp. 2338-2353.

[7] M. Dong and L. Tong, "Optimal design and placement of pilot symbols for channel estimation," IEEE Trans. Signal Processing, Vol. 50, No. 12, Dec. 2002, pp. 3055-3069.

[8] S. Ohno and G. B. Giannakis, "Optimal Training and Redundant Precoding for Block Transmissions with Application to Wireless OFDM," IEEE Trans. on Comun., Vol. 50, No. 12, Dec. 2002, pp. 2113-2123.

[9] X. Ma, G.B. Giannakis and S. Ohno, "Optimal training for block transmissions over doubly selective wireless fading channels," IEEE Trans. Signal Processing, Vol. 51, No. 5, May 2003, pp. 1351-1365.

[10] C. Fragouli, N. Al-Dhahir, and W. Turin, "Training-Based Channel Estimation for MultipleAntenna Broadband Transmissions," IEEE Trans. on Wireless Commun., Vol. 2, No. 2, Mar. 2003, pp. 384-391.

[11] I. Barhumi, G. Leus, and M. Moonen, "Optimal Training Design for MIMO OFDM Systems in Mobile Wireless Channels," IEEE Trans. on Signal Processing, Vol. 51, No. 6, June 2003, pp. 1615-1624.

[12] Y. Li, "Simplified Channel Estimation for OFDM Systems with Multiple Transmit Antennas," IEEE Trans. on Wireless Communications, Vol. 1, No. 1, Jan. 2002, pp. 67-75.

[13] S. M. Kay, "Fundamentals of Statistical Signal Processing: Estimation Theory," Prentice Hall PTR, 1993.
TABLE II

An Optimal Pilot Allocation among $N_{\text {Tx }}$ TRAnsmit antennas in AN OFDM SYSTEM WITH $K=16$ SUB-CARRIERS OVER $Q=2$ OFDM SYMBOLS FOR ESTIMATION OF CHANNELS WITH $L=4$ TAPS EACH (ANTENNAS ASSIGNMENT ON THE GRID OF SUB-CARRIERS AND SYMBOLS )

\begin{tabular}{||c|c|c||}
\hline \multicolumn{3}{|c||}{ Antennas assignments } \\
\hline $\begin{array}{c}\text { Sub-carrier } \\
\text { Index }\end{array}$ & \multicolumn{2}{|c|}{ Symbol Index } \\
\hline 0 & $0,1,2,3$ & $0,1,2,3$ \\
\hline 1 & $0,1,2,3$ & $0,1,2,3$ \\
\hline 2 & 4 & 6,7 \\
\hline 3 & 5 & 6,7 \\
\hline 4 & $0,1,2,3$ & $0,1,2,3$ \\
\hline 5 & $0,1,2,3$ & $0,1,2,3$ \\
\hline 6 & 4 & 6,7 \\
\hline 7 & 5 & 6,7 \\
\hline 8 & $0,1,2,3$ & $0,1,2,3$ \\
\hline 9 & $0,1,2,3$ & $0,1,2,3$ \\
\hline 10 & 6,7 & 4 \\
\hline 11 & 5 & 6,7 \\
\hline 12 & $0,1,2,3$ & $0,1,2,3$ \\
\hline 13 & $0,1,2,3$ & $0,1,2,3$ \\
\hline 14 & 6,7 & 4 \\
\hline 15 & 5 & 6,7 \\
\hline \multicolumn{3}{|c|}{} \\
\hline
\end{tabular}

\section{TABLE III}

Optimal Pilot Tone Vectors For $N_{\text {Tx }}=8$ transmit antenNas IN AN OFDM SYSTEM WITH $K=16$ SUB-CARRIERS OVER $Q=2$ OFDM SYMBOLS FOR ESTIMATION OF CHANNELS WITH $L=4$ TAPS EACH

\begin{tabular}{|c|c|c|c|c|c|c|c|c|}
\hline \multirow[b]{2}{*}{$q$} & \multicolumn{8}{|c|}{ Antenna Index } \\
\hline & 0 & 1 & 2 & 3 & 4 & 5 & 6 & 7 \\
\hline \multirow{16}{*}{0} & $\alpha_{1}$ & $\alpha_{1}$ & $\alpha_{1}$ & $\alpha_{1}$ & 0 & 0 & 0 & 0 \\
\hline & $\alpha_{2}$ & $-\alpha_{2}$ & $\alpha_{2}$ & $-\alpha_{2}$ & 0 & 0 & 0 & 0 \\
\hline & 0 & 0 & 0 & 0 & $2 \alpha_{9}$ & 0 & 0 & 0 \\
\hline & 0 & 0 & 0 & 0 & 0 & $2 \alpha_{13}$ & 0 & 0 \\
\hline & $\alpha_{3}$ & $\alpha_{3}$ & $\alpha_{3}$ & $\alpha_{3}$ & 0 & 0 & 0 & 0 \\
\hline & $\alpha_{4}$ & $-\alpha_{4}$ & $\alpha_{4}$ & $-\alpha_{4}$ & 0 & 0 & 0 & 0 \\
\hline & 0 & 0 & 0 & 0 & $2 \alpha_{10}$ & 0 & 0 & 0 \\
\hline & 0 & 0 & 0 & 0 & 0 & $2 \alpha_{14}$ & 0 & 0 \\
\hline & $\alpha_{5}$ & $\alpha_{5}$ & $\alpha_{5}$ & $\alpha_{5}$ & 0 & 0 & 0 & 0 \\
\hline & $\alpha_{6}$ & $-\alpha_{6}$ & $\alpha_{6}$ & $-\alpha_{6}$ & 0 & 0 & 0 & 0 \\
\hline & 0 & 0 & 0 & 0 & 0 & 0 & $\sqrt{2} \alpha_{17}$ & $\sqrt{2} \alpha_{17}$ \\
\hline & 0 & 0 & 0 & 0 & 0 & $2 \alpha_{15}$ & 0 & 0 \\
\hline & $\alpha_{7}$ & $\alpha_{7}$ & $\alpha_{7}$ & $\alpha_{7}$ & 0 & 0 & 0 & 0 \\
\hline & $\alpha_{8}$ & $-\alpha_{8}$ & $\alpha_{8}$ & $-\alpha_{8}$ & 0 & 0 & 0 & 0 \\
\hline & 0 & 0 & 0 & 0 & 0 & 0 & $\sqrt{2} \alpha_{18}$ & $\sqrt{2} \alpha_{18}$ \\
\hline & 0 & 0 & 0 & 0 & 0 & $2 \alpha_{16}$ & 0 & 0 \\
\hline \multirow{16}{*}{1} & $\alpha_{1}$ & $\alpha_{1}$ & $-\alpha_{1}$ & $-\alpha_{1}$ & 0 & 0 & 0 & 0 \\
\hline & $\alpha_{2}$ & $-\alpha_{2}$ & $-\alpha_{2}$ & $\alpha_{2}$ & 0 & 0 & 0 & 0 \\
\hline & 0 & 0 & 0 & 0 & 0 & 0 & $\sqrt{2} \alpha_{19}$ & $\sqrt{2} \alpha_{19}$ \\
\hline & 0 & 0 & 0 & 0 & 0 & 0 & $\sqrt{2} \alpha_{19}$ & $-\sqrt{2} \alpha_{19}$ \\
\hline & $\alpha_{3}$ & $\alpha_{3}$ & $-\alpha_{3}$ & $-\alpha_{3}$ & 0 & 0 & 0 & 0 \\
\hline & $\alpha_{4}$ & $-\alpha_{4}$ & $-\alpha_{4}$ & $\alpha_{4}$ & 0 & 0 & 0 & 0 \\
\hline & 0 & 0 & 0 & 0 & 0 & 0 & $\sqrt{2} \alpha_{20}$ & $\sqrt{2} \alpha_{20}$ \\
\hline & 0 & 0 & 0 & 0 & 0 & 0 & $\sqrt{2} \alpha_{20}$ & $-\sqrt{2} \alpha_{20}$ \\
\hline & $\alpha_{5}$ & $\alpha_{5}$ & $-\alpha_{5}$ & $-\alpha_{5}$ & 0 & 0 & 0 & 0 \\
\hline & $\alpha_{6}$ & $-\alpha_{6}$ & $-\alpha_{6}$ & $\alpha_{6}$ & 0 & 0 & 0 & 0 \\
\hline & 0 & 0 & 0 & 0 & $2 \alpha_{11}$ & 0 & 0 & 0 \\
\hline & 0 & 0 & 0 & 0 & 0 & 0 & $\sqrt{2} \alpha_{21}$ & $-\sqrt{2} \alpha_{21}$ \\
\hline & $\alpha_{7}$ & $\alpha_{7}$ & $-\alpha_{7}$ & $-\alpha_{7}$ & 0 & 0 & 0 & 0 \\
\hline & $\alpha_{8}$ & $-\alpha_{8}$ & $-\alpha_{8}$ & $\alpha_{8}$ & 0 & 0 & 0 & 0 \\
\hline & 0 & 0 & 0 & 0 & $2 \alpha_{12}$ & 0 & 0 & 0 \\
\hline & 0 & 0 & 0 & 0 & 0 & 0 & $\sqrt{2} \alpha_{22}$ & $-\sqrt{2} \alpha_{22}$ \\
\hline
\end{tabular}

